\title{
Cycles in Public Opinion and the Dynamics of Stable Party Systems
}

\author{
Sandro Brusco ${ }^{\S} \quad$ Jaideep Roy
}

October 1, 2015

\begin{abstract}
We study a dynamic model of elections where many parties may enter or exit political competition. At each election a new political leadership arrives for each party. The leadership cannot choose the party's platform (ideological identities are fixed) but must decide whether or not to contest the election. Contesting elections is costly and this cost is higher if the party has recently been inactive. The distribution of voters ideal policies, or public opinion, changes over time via a Markov process with a state independent persistence parameter. We characterise stable party systems where the set of contestants is invariant to the recent most observed opinion. We show that stable party systems exist only when public opinion is sufficiently volatile, while highly persistent moods lead to instability and change in the party system whenever public opinion changes.
\end{abstract}

Keywords: Public Opinion, Aggregate Uncertainty, Party Systems.

JEL Classification: D72.

\section{Introduction}

The impermanence of public opinion is well documented in political science. ${ }^{1}$ As put succinctly by Stimson [18], "we expect it to cycle back and

\footnotetext{
${ }^{\S}$ Department of Economics, Stony Brook University, USA. E-mail: sandro.brusco@stonybrook.edu.

IDepartment of Economics, Deakin University, Melbourne, Australia; Email: j.roy@deakin.edu.au.

${ }^{1}$ See the special issue of Electoral Studies $(19,2000)$ for a large literature on this.
} 
forth, left and right, as leaders and followers change their views of government policy over time." This precariousness of public opinion, according to Schlesinger [21], is likely to be grounded in people's inability to remain fulfilled for long and as political eras run their course, they "infallibly generate the desire for something different." Since public opinion shapes social cleavages and determines the ideology of decisive voters, one would expect political outcomes to stagger along with the time dynamics of public opinion where ideologically new parties take the centre stage while some established parties cease to dominate for their inability to rapidly change ideological identities. Yet, for close to a century now, Western democracies have exhibited a remarkable continuity and stability in their party systems, both in terms of number of contestants and their ideological positions (see Bartolini and Mair [3]). At the same time, the appearance of new parties and the decamping of the established ones are also not rare. ${ }^{2}$

This raises a challenging question that has been largely ignored: what makes party systems stable when public opinion is inherently volatile and when do stable party systems break down? The contribution of this paper is to endogenize the entry and exit decisions and analyse the implications of different stochastic structures for public opinion on the stability and evolution of party systems.

Most formal theories on the entry and exit of political parties are static (for example Feddersen et al. [11] and Greenberg and Shepsle [12]) and do not deal explicitly with the changes in party systems induced by an ever-changing public opinion. Formal dynamic models of the evolution of the party system have recently appeared in the literature but, to the best of our knowledge, they have either assumed that the number of parties is fixed (usually to two) or have assumed random processes for entry (a recent survey of the results is in Duggan and Martinelli [10]). Merrill et al. [15] provides statistical evidence for the existence of cycles in American politics and propose a simple adaptive model in which the two main parties change their position adaptively over time as the median voter changes positions. However, they do not consider entry and exit and they do not provide 'microfoundations' for party behaviour. Duggan and Forand [9] study a model with a finite set of voters in which the political state (that includes public opinion in terms of the location of the set of decisive voters) changes over time via a Markov process. In their model, a potential candidate lives for ever and is available for selection unless as an incumbent he withdraws from the contest or gets defeated by a random challenger, in which case he exits the political arena forever. Also, elections

\footnotetext{
${ }^{2}$ For example, Hug [13] finds that in 22 major Western democracies as many as 361 new parties have formed since 1945 .
} 
are always a two-candidate contest, one being the incumbent party (who has the option of withdrawing from the race) and the other being a random challenger with unknown type. Our paper instead studies large elections (where there are a continuum of voters) and explicitly deals with the entry and exit decisions of parties. We analyse a model in which established parties have a structural advantage (they do not have to pay the 'entry cost') but at every period there are multiple potential entrants who may decide strategically to challenge the existing parties or stay out. Also, party identities live for ever, though the leadership has a short-term horizon.

We develop a dynamic theory of first-past-the-post elections based on two dominant stylised facts of a competitive democracy. First, both party entry and permanence over time are assumed to be costly. As shown in Abramson et al. [1] for the case of American politics, new entry in the political arena has many barriers favouring established parties. These barriers exist in one form or another in any competitive democracy and a new party must pay a cost to overcome them in the first place. Besides entry, running a party - new or well established - involves operating costs in each period. Thus, costs incurred by parties in our model have two components, namely, an entry cost and a running cost. It implies that at any period, the total cost of participating in an election is strictly higher when the party is new.

Second, public opinion, modelled as the distribution of the voters' ideal points on a one dimensional policy space, changes over time with an element of randomness and yet exhibiting a certain degree of persistence. The existence of cycles in public opinion has been recognised for a long time. Stimson ([18], [19], [20]) documents how the attitudes of the American public on various issues tend to move over time. Besides, opinions on different issues tend to correlate quite strongly, so that it makes sense to adopt the simplifying assumption that the ideology/policy space is single dimensional. On the other hand, Byers and Peel [7], DeBoef [8], Lebo et al. [14] and Wlezien [24] show that an important feature of political time series is persistence. We therefore model the evolution of political mood as a Markov process that is determined by a single parameter representing 'persistence' - the probability that public opinion will remain the same in the 'next election'. We assume that each current public opinion continues to the next period with this probability and otherwise changes to a new one from a fixed finite set of possible opinions.

Another important and novel feature of our model is motivated by some very strong empirical evidence on the fact that a party's ideology remains more or less fixed over time. For example, Budge [6] and Adam et al. [2] show that parties exhibit great reluctance in changing their ideological identities. We model this by assuming that there is a given set of potentially active parties identified by ideological stands that cannot be altered by new 
leaders who arrive afresh in each period. Thus, while newly appointed leaders decide whether to compete (and thus be active in our sense) or not during their leadership tenure, their only credible policy platform remains fixed to the given party ideology. This is similar to the Citizen-Candidate models of Osborne and Slivinski [16] and Besley and Coate [4] but with an important distinction. In their models, politicians have strong ideological biases that bereave them from their ability to make credible policy commitments different from their ideal points. In our case, politicians are not 'ideologues' in that sense as all they care about is winning elections. However their party affiliation constrains them from choosing electoral platforms freely, unlike in the case of the standard Downsian paradigm.

We also take into account the fact that while many elections have been tightly fought, an exactly tied verdict in any large election has never been observed. To that end, we assume that under each feasible political mood, there is always a single winner (the identity of the winner depends on the location of the competitors). This then immediately implies that multiple party contests must be the result of unresolved aggregate uncertainty about public mood.

Given this environment, a party system is stable if, independently of the true public mood in the immediately held elections, each party finds it strictly profitable to continue contesting and no new party finds it profitable to enter. Since in each period each party has a new leader, stability implies that in spite of the fact that public mood can change, each active party expects to win with sufficiently high probability to cover the costs of running in the next elections.

We show that in order to have a stable multi-party system, persistence of public opinion cannot be too high - that is, public opinion must be sufficiently volatile. This is due to the fact that a high degree of persistence invites exit of defeated parties. We characterise a two-party stable system. Apart from the conditions on persistence mentioned above, we report those that are required on the exact nature of the distributions that are necessary and sufficient to obtain the Duverger's Law of a two-party system that is in addition stable. These requirements yield a refinement of the conditions found in Brusco and Roy [5], where a static citizen-candidate model is studied under aggregate uncertainty. We then study long run dynamics of a stable multi-party system and show that stability will typically be impaired when the political mood does not change and the same party keeps winning elections. In that case at some point in the future, either some of the losers will exit or some entry will occur.

The rest of the paper is organised as follows. Section 2 lays out the basic elements of the model. In Section 3 we discuss the equilibrium concept and 
characterise stable party systems. In Section 4 we address long run outcomes and dynamics. Section 5 discusses an example with political moods having normal distributions. Conclusions are in Section 6 and the proofs are in an Appendix.

\section{The Model}

Elections are held at each period $t=0,1, \ldots$. There is a finite set $P=$ $\{1, \ldots, r\}$ of (potential) political parties. Each party $i$ has a fixed identity given by a point $x_{i}$ on the ideology-policy line $\mathbb{R}$. The set $X=\left\{x_{1}, \ldots, x_{r}\right\}$ is thus called the set of feasible policies. Parties are labeled so that $x_{1}<$ $x_{2}<\ldots<x_{r}$.

Parties are 'run' by political leaders (or, simply, politicians) who live for a single period. A politician who becomes the leader of party $i \in P$ must adopt the ideology-policy platform $x_{i}$ given by party $i$ 's identity. While politicians are constrained to adopt the fixed identity of their parties, they can decide whether or not to contest the election under the party banner. Thus, at each time $t$ a game of entry is played among $r$ new politicians, each of them having action set $A_{i}=\{0,1\}$, where $a_{i}=1$ means that party $i$ is contesting the elections with platform $x_{i}$ while $a_{i}=0$ means that the party opts out of the current electoral competition. An action profile $\mathbf{a}^{t}=$ $\left\{a_{1}^{t}, \ldots, a_{r}^{t}\right\} \in\{0,1\}^{r}$ determines the set of parties contesting at time $t$. Let $P\left(\mathbf{a}^{t}\right)=\left\{i \in P \mid a_{i}^{t}=1\right\}$ be the set of active parties when the action profile is $\mathbf{a}^{t}$. At each election a party is chosen according to the first-past-the-post voting rule.

\subsection{Political Moods}

There is a continuum of voters and in each period $t$ their preferences are described by a measure $\mu^{t}$ over $\mathbb{R}$, which we call the political mood. Given a political mood, voters vote sincerely, i.e. they vote for the party with identity closest to their ideal point. The political mood evolves stochastically over time. We model this change assuming there is a finite state space $\mathcal{M}=\left\{\mu_{1}, \ldots, \mu_{n}\right\}$ of possible measures on $\mathbb{R}$ and denote with $m_{i}$ the median of distribution $\mu_{i} \in \mathcal{M}$ and with $F_{i}$ the cumulative distribution function. Distributions are labeled in order of increasing medians, i.e. $m_{1}<m_{2}<\ldots<m_{n}$.

At each period $t$ the measure $\mu^{t}$ is randomly selected from $\mathcal{M}$. The 
probability distribution over $\mathcal{M}$ is described by the Markov process:

$$
\operatorname{Pr}\left(\mu^{t}=\mu_{j} \mid \mu^{t-1}=\mu_{i}\right)=\left\{\begin{array}{ccc}
\theta^{t} & \text { if } j=i \\
\frac{1-\theta^{t}}{n-1} & \text { if } j \neq i .
\end{array}\right.
$$

Thus, $\theta^{t}$ measures the degree of persistence of a political mood at time $t$ and it belongs to a subset $\Theta$ of the interval $[0,1]$. We assume that when the political mood changes, any of the 'other' moods is equally likely to be selected, but the assumption is only made for simplicity and can easily be weakened at the cost of complicating notation. The important feature of the assumption is that the probability of not changing the political mood is the same for each current political mood. We make the following assumption.

Assumption 1 (Unique winner). For each entry vector $\mathbf{a} \in\{0,1\}^{r}$ with at least one $a_{i}=1$ and for each measure $\mu_{j} \in \mathcal{M}$ the election has a unique winner, denoted by $w\left(\mathbf{a}, \mu_{j}\right)$. For each $i \in\{1, \ldots, r\}$ and $j \in\{1, \ldots, n\}$ we have $x_{i} \neq m_{j}$. If no one enters then the policy implemented is a default policy $x^{o} \in \mathbb{R}$.

The assumption is actually a collection of different sets of assumptions, one for each $\mu_{j} \in \mathcal{M}$. Essentially it states that points in the set of feasible policies $X$ are positioned in a way that, for any given non-empty subset of them and for each possible measure $\mu_{j} \in \mathcal{M}$, there are no ties with respect to vote shares and no political platform coincides with the median of any distribution (this is only needed to simplify calculations later and can be dispensed). It is straightforward to show that violating Assumption 1 leads to non-genericity of the environment under study.

\subsection{Payoffs}

Party leaders are purely office-seeking and they decide whether to contest the election in order to maximise the difference between expected benefits and costs. The utility of party $j$ when the past action vector is $\mathbf{a}^{t-1}$, the current action vector is $\mathbf{a}^{t}$ and the current political mood is $\mu_{i}$ is given by

$$
u_{j}\left(\mathbf{a}^{t}, \mu_{i}, a_{j}^{t-1}\right)=\left\{\begin{array}{cc}
b-a_{j}^{t}\left(c_{p}+\left(1-a_{j}^{t-1}\right) c_{e}\right) & \text { if } w\left(\mathbf{a}^{t}, \mu_{i}\right)=j \\
-a_{j}^{t}\left(c_{p}+\left(1-a_{j}^{t-1}\right) c_{e}\right) & \text { otherwise }
\end{array}\right.
$$

where $b>0$ is the benefit from winning office, $c_{p}>0$ is the cost of contesting the election and $c_{e}>0$ is the additional entry cost to be paid if the party 
was out of competition in the previous period. ${ }^{3}$

We normalize the benefits from office and set $b=1$. This means that costs are interpreted as fraction of benefits from office holding. To make the problem interesting we assume $c_{e}+c_{p}<1$ as otherwise no new entry can be obtained in this model.

Utility over mixed strategies is defined in the standard way. If $\boldsymbol{\sigma}_{-j}\left(\mathbf{a}_{-j}\right)=$ $\prod_{i \neq j} \sigma_{i}\left(a_{i}\right)$ is the probability of action profile $\mathbf{a}_{-j}$, then the expected utility of a period $t$ politician from action $a_{j}$ is

$$
u_{j}\left(\left(a_{j}, \boldsymbol{\sigma}_{-j}\right), \mu_{i}, a_{j}^{t-1}\right)=\sum_{\mathbf{a}_{-j} \in A_{-j}} \boldsymbol{\sigma}_{-j}\left(\mathbf{a}_{-j}\right) u_{j}\left(\left(a_{j}, \mathbf{a}_{-j}\right), \mu_{i}, a_{j}^{t-1}\right) .
$$

Finally, we can define the expected utility when $\mu_{i}$ is unknown. If $\psi_{i}$ is the probability given to $\mu_{i} \in \mathcal{M}$ then

$$
\mathbf{E}_{\psi}\left[u_{j}\left(\left(a_{j}, \boldsymbol{\sigma}_{-j}\right), \mu_{i}, a_{j}^{t-1}\right)\right]=\sum_{i=1}^{n} \psi_{i} u_{j}\left(\left(a_{j}, \boldsymbol{\sigma}_{-j}\right), \mu_{i}, a_{j}^{t-1}\right) .
$$

Since the game has a finite set of pure strategies and a finite set of players in each period $t$, a Nash equilibrium in mixed strategies exists for each probability distribution $\psi$ and profile $\mathbf{a}^{t-1}$ of actions taken in the previous period.

\subsection{Information and Beliefs}

At each period $t$, politicians decide whether to contest or not before observing the realisation of the state $\mu^{t}$; they only know the history of political configurations $\left\{\mathbf{a}^{\tau}\right\}_{\tau=1}^{t-1}$ and the history of political moods up to the previous period $\left\{\mu^{\tau}\right\}_{\tau=0}^{t-1}$. Since it is common knowledge that the probability distribution on $\mathcal{M}$ is given by (1) and $\mu^{t-1}$ is observed, the expected utility of a period $t$ politician representing party $j$ can be defined as

$$
\begin{gathered}
V_{j}\left(\left(a_{j}, \boldsymbol{\sigma}_{-j}\right), a_{j}^{t-1} \mid \mu_{k}, \theta^{t}\right)= \\
\theta^{t} u_{j}\left(\left(a_{j}, \boldsymbol{\sigma}_{-j}\right), \mu_{k}, a_{j}^{t-1}\right)+\left(1-\theta^{t}\right) \frac{\sum_{i \neq k} u_{j}\left(\left(a_{j}, \boldsymbol{\sigma}_{-j}\right), \mu_{i}, a_{j}^{t-1}\right)}{n-1} .
\end{gathered}
$$

\footnotetext{
${ }^{3}$ If party $j$ also cared about policies, then one would add the term $v_{j}\left(w\left(\mathbf{a}^{t}, \mu_{i}\right)\right)$ to the expression for $u_{j}\left(\mathbf{a}^{t}, \mu_{i}, a_{j}^{t-1}\right)$ where $v_{j}(i)$ gives the utility of the party $j$ when policy $x_{i} \in X$ is implemented.
} 
When there is uncertainty over $\theta^{t}$, at the beginning of time $t$, the players' beliefs on $\theta^{t}$ can be represented by a probability distribution $\phi^{t}$, with the distribution typically dependent on the observed history.

We assume that $\theta^{t}$ can take a finite number of values from the set $\Theta=$ $\left\{\theta_{1}, \ldots, \theta_{m}\right\}$ and denote by $\phi_{k}$ the probability of $\theta_{k}$. Let $\theta_{e}^{\phi}=\sum_{k=1}^{n} \phi_{k} \theta_{k}$ be the expected value of $\theta$ under distribution $\phi$. Incorporating this higher order uncertainty, it is easy to see that $\theta_{e}^{\phi}$ is the only relevant moment of the distribution, since the expected utility of party $j$ is given by

$$
\begin{gathered}
\mathbf{V}_{j}\left(\phi,\left(a_{j}, \boldsymbol{\sigma}_{-j}\right), a_{j}^{t-1}, \mu_{k}\right)=\sum_{q=1}^{m} \phi_{q} V_{j}\left(\left(a_{j}, \boldsymbol{\sigma}_{-j}\right), a_{j}^{t-1} \mid \mu_{k}, \theta_{q}\right)= \\
\theta_{e}^{\phi} u_{j}\left(\left(a_{j}, \boldsymbol{\sigma}_{-j}\right), \mu_{k}, a_{j}^{t-1}\right)+\left(1-\theta_{e}^{\phi}\right) \frac{\sum_{i \neq k} u_{j}\left(\left(a_{j}, \boldsymbol{\sigma}_{-j}\right), \mu_{i}, a_{j}^{t-1}\right)}{n-1} .
\end{gathered}
$$

Thus, the expected utility depends linearly on $\theta_{e}^{\phi}$ and the best response is always the pure strategy $a_{i} \in\{0,1\}$ when $\theta_{e}^{\phi}$ is below a certain threshold and the 'opposite' pure strategy $1-a_{i}$ when that threshold is crossed.

\section{Stable Party Systems}

One of the most robust features of any well-functioning democracy is perhaps that the existing group of major political parties is remarkably stable: they sometimes win and sometime lose elections depending on the political mood, but each of them tend to stay put and do not instantly disappear after a defeat. At the same time we do observe occasional births and deaths of parties, although these events are relatively rare. To understand these characteristics of an evolving democracy, two things need to be addressed. First, we need to understand conditions under which stability of party systems is the outcome of equilibrium behaviour in a model like the one we are proposing; this is the focus of this section. After that we need to understand how a stable system is sustained in the long run or is disturbed so that democracies transit from one stable system to another. We shall address long run dynamics in Section 4.

\subsection{Definition}

Politicians in our model live for one period and therefore at each election the entry game is a one-shot game with aggregate uncertainty. So the actions of the politicians in each period should form a Nash equilibrium of the oneshot game. However, at time $t$ the vector $\mathbf{a}^{t-1}$ of actions undertaken in the 
previous period determines the cost of contest for each politician. Furthermore, if there is uncertainty over the persistence of the political mood, then history is used to determine the probability distribution $\phi^{t}$ on $\Theta$. With this clarification, the following definition is standard.

Definition 1 (Equilibrium). For a given probability distribution $\phi$ on $\Theta$, political mood $\mu_{k}$ and vector $\mathbf{a}^{t-1}$ of political activities in the previous period, a Nash equilibrium at time $t$ is a probability distribution $\boldsymbol{\sigma}$ such that

$$
\mathbf{V}_{j}\left(\phi,\left(a_{j}, \boldsymbol{\sigma}_{-j}\right), a_{j}^{t-1}, \mu_{k}\right) \geq \mathbf{V}_{j}\left(\phi,\left(1-a_{j}, \boldsymbol{\sigma}_{-j}\right), a_{j}^{t-1}, \mu_{k}\right)
$$

for each $a_{j}$ such that $\sigma_{j}\left(a_{j}\right)>0$. When the inequalities are strict for each participant then we say that the Nash equilibrium is strict.

A strict Nash equilibrium can only be in pure strategies and, as previously observed, the only moment of the distribution $\phi$ that matters is the mean $\theta_{e}^{\phi}$. The mean $\theta_{e}^{\phi}$ can take any value in the interval $\left[\theta_{1}, \theta_{m}\right]$. We can now provide our definition of stable party system.

Definition 2 (Stable Party System). A stable party system is a pair $\left(\mathbf{a}^{*}, \Theta^{*}\right)$ where $\Theta^{*} \subset\left[\theta_{1}, \theta_{m}\right]$ such that for each $\mu \in \mathcal{M}$ and for each distribution $\phi$ such that $\theta_{e}^{\phi} \in \Theta^{*}$, the vector $\mathbf{a}^{*}$ is a strict Nash equilibrium when the entry vector of the previous period was $\mathbf{a}^{*}$.

In other words, as long as the expected value of the persistence parameter remains in a certain set $\Theta^{*}$, the same set of parties will keep contesting the election no matter what the result of the last election (determined by $\mu$ ) was. A stable party system can only be overcome when there is a significant change in the perceived degree of persistence, i.e. when the expected value $\theta_{e}$ exits out of the set $\Theta^{*}$. $^{4}$

\subsection{Characterisation and Existence}

The first thing to observe about stable party systems is that, given the requirement that the action profile remains a strict Nash equilibrium in each period and the 'threshold property' of the best responses, the set $\Theta^{*}$ must be an interval.

Proposition 1. In each stable party system $\left(\mathbf{a}^{*}, \Theta^{*}\right)$ the set $\Theta^{*}$ is an interval.

\footnotetext{
${ }^{4}$ In entry games it is typical to have multiple equilibria. One source of instability for the political system could come simply from the fact that in different periods, even when the fundamentals do not change, different equilibria are selected. We disregard this potential source of instability and assume that, whenever possible, the equilibrium realised at time $t-1$ will serve as a focal point at period $t$.
} 
Proposition 1 follows immediately from the fact that expected utilities are linear in $\theta_{e}$. If a vector $\mathbf{a}^{*}$ is a strict Nash equilibrium when the previous distribution was $\mu_{k}$ for two values of $\theta_{e}$, then all linear combinations must preserve the same inequalities that define a strict Nash equilibrium. The set $\Theta^{*}$ that defines a stable party system is then given by the intersection of all the intervals, if it's not empty.

The proposition however says nothing about existence. In order to find conditions for existence of stable party systems, we look first at the existence of Nash equilibria. Entry can be optimal only if the probability of victory is strictly positive, so Assumption 1 implies that in a pure strategy equilibrium the number of parties cannot exceed the number of states. Furthermore, let $i_{k}=w\left(\mu_{k}, \mathbf{a}\right)$ be the unique winner under distribution $\mu_{k}$ and entry vector $\mathbf{a}$. If $\mu_{k}$ is indeed the realised mood in the immediate past, then the expected utility $V_{i_{k}}$ of party $i_{k}$ in the current period is strictly increasing in $\theta_{e}$. The expected utility is instead decreasing in $\theta_{e}$ for $j \neq i_{k}$. Proposition 2 shows that when $\theta_{e}$ crosses a threshold, any pure strategy equilibria can have at most one party and that the active party is the closest to the median $m_{k}$ of the distribution realised in the immediate history.

Proposition 2. Suppose $\theta_{e}>1-c_{p}$ and the realized distribution in period $t-1$ is $\mu_{k}$. Then in every pure strategy equilibrium only one party enters. Furthermore, if $\theta_{e}>\max \left\{1-c_{p}, c_{p}+c_{e}\right\}$, the equilibrium is unique and the only active party is the one closest to $m_{k}$.

The logic behind Proposition 2 is simple. When $\theta_{e}$ is close to one, it means that the perceived probability that the state $\mu_{k}$ will persist is close to one. Thus, any politician who does not forecast to win under $\mu_{k}$ will prefer to stay out. This gives us the bound $1-c_{p}$. Given Assumption 1 at most one party can enter, and if that party is not the one closest to the median $m_{k}$, then profitable entry becomes possible when persistence is large enough to cover the entry cost in addition to the running cost. This gives us the bound $c_{p}+c_{e}$. Thus, when $\theta_{e}$ is high then one-party equilibria are not sustainable unless the party is the closest to the median. Hence, when the perceived persistence is higher than these two bounds, then the unique outcome is that the most popular party under $\mu_{k}$ is the uncontested winner, a 'median voter' result.

Proposition 2 also implies that when $\theta_{e}$ is high then the strict Nash equilibrium is necessarily different for each distribution, and so there is no vector $\mathbf{a}^{*}$ that can remain an equilibrium under different distributions. This leads to the following corollary.

Corollary 1. There is no stable party system $\left(\mathbf{a}, \Theta^{*}\right)$ such that $\theta_{e} \in \Theta^{*}$ and $\theta_{e}>\max \left\{1-c_{p}, c_{p}+c_{e}\right\}$. 
Stable party systems are therefore possible only if $\theta_{e}$ is not too high. We now proceed to investigate condition for existence. ${ }^{5}$

\subsection{Stable One-Party Systems}

A stable one-party system in our framework is akin to the existence of a single dominant party that finds it profitable to continue its regular activities irrespective of what the current public mood is. There are some instances where well-functioning democracies experienced something close to a one party system. For example, since the inception of parliamentary democracy in India, the Indian National Congress has been the dominant political party and had been in power from 1946 to 1989 almost unopposed.

We first establish necessary and sufficient conditions for the existence of a stable one party system. In this case the current active party wins with probability 1 and has no unilateral incentives to exit. So the only thing that needs to be satisfied is entry prevention. Proposition 3 provides tight conditions for the existence of a one party system. We use the notation $\lfloor x\rfloor$ to denote the integer part of $x$.

Proposition 3. Let $n$ be the cardinality of $\mathcal{M}$. The necessary and sufficient condition for the existence of a stable one party system is $c_{e}+c_{p}>\frac{1}{n} \times\left\lfloor\frac{n-1}{2}\right\rfloor$. In a stable one party system $\left(\mathbf{a}^{*}, \Theta^{*}\right)$ the set $\Theta^{*}$ takes either the form $\Theta^{*}=$ $\left[0, \theta^{*}\right)$ or $\left(\theta_{*}, \theta^{*}\right)$ with $\theta^{*}>\frac{1}{n}>\theta_{*}$.

There may be multiple stable one party systems. The equilibria which are most 'robust', in the sense that they exist for 'most' values of $c_{e}+c_{p}$ satisfying the conditions of Proposition 3, are the ones in which the incumbent is located in the centre of the political spectrum, i.e. in the interval $\left(m_{\left\lfloor\frac{n}{2}\right\rfloor}, m_{\left\lfloor\frac{n}{2}\right\rfloor+1}\right)$.

That a sufficiently high value of $c_{e}+c_{p}$ is needed to support a one-party equilibrium is obvious. The intuition on why we need small values of $\theta$ is the same as in Corollary 1. In a one party system at some point there are mood realisations which are 'far' from where the party is located. If there is a sufficiently high probability that the mood persists then entry becomes optimal and a one party system cannot be stable.

\footnotetext{
${ }^{5}$ It is useful to observe that a symmetric result for the case in which $\theta_{e}$ is very low does not apply. To see this, suppose $\theta_{e}=0$. Then it is certain that the mood $\mu$ will not be the last observed mood $\mu_{k}$, while all other moods will appear with probability $\frac{1}{n-1}$. However, the party winning under $\mu_{k}$ does not necessarily have a zero probability of winning when the mood changes, since it may win under other distributions. This is in contrast to the case in which $\theta_{e}=1$ where only one party can win.
} 


\subsection{Stable Multi-Party Systems}

All mature democracies today have established multi-party systems. When the party system has multiple active parties, realisation and persistence of public opinion start playing a more involved role than with a single dominant party as conditions to prevent exit of established parties gain importance. The very fact that a multi-party system exists in a world of office-seeking politics implies each active party wins with certainty under some realisations of public mood. Thus high persistence can quickly throw current losers out of contest while low persistence can force current winners to become inactive.

To be precise, let $\mathbf{a}^{*}$ be an entry vector and define $q_{i}\left(\mathbf{a}^{*}\right)$ as the number of moods under which party $i$ wins. Stability requires that each incumbent party must be willing to stay both when it is winning under the current distribution and when it is not. Thus, for each $i \in P\left(\mathbf{a}^{*}\right)$ it has to be the case that

$$
\min \left\{\theta+(1-\theta) \frac{q_{i}\left(\mathbf{a}^{*}\right)-1}{n-1},(1-\theta) \frac{q_{i}\left(\mathbf{a}^{*}\right)}{n-1}\right\}>c_{p}
$$

The maximum value of the LHS is attained at $\theta=\frac{1}{n}$, so a necessary condition for $\mathbf{a}^{*}$ to be a stable party system is

$$
\min _{i \in P\left(\mathbf{a}^{*}\right)} \frac{q_{i}\left(\mathbf{a}^{*}\right)}{n} \geq c_{p}
$$

To have a better sense of the conditions for existence, consider the party $i^{*} \in P\left(\mathbf{a}^{*}\right)$ such that $q_{i^{*}}\left(\mathbf{a}^{*}\right)$ is the lowest. If it is in fact the case that $\frac{q^{*}\left(\mathbf{a}^{*}\right)}{n}>c_{p}$, then the set of values of $\theta$ for which no incumbent is willing to leave can be seen by drawing the left hand side of inequality (4) as a function of $\theta$.
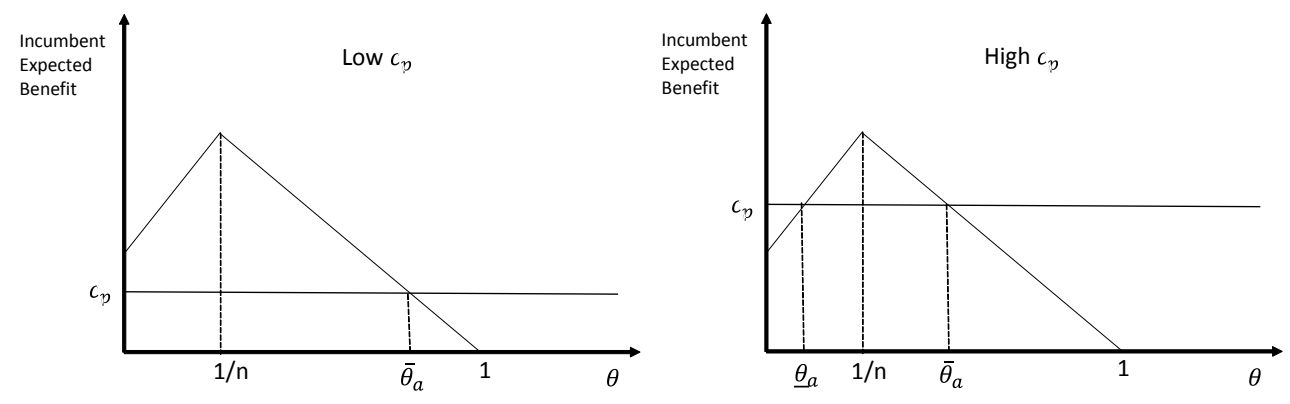

When the value of $c_{p}$ is sufficiently low $\left(c_{p}<\frac{q_{i^{*}-1}}{n-1}\right)$ then the interval of values of $\theta$ for which all incumbent parties are willing to stay is $\left[0, \bar{\theta}_{a}\right)$. When the value of $c_{p}$ is higher $\left(\frac{q_{i *}-1}{n-1} \leq c_{p}<\frac{q_{i^{*}}}{n}\right)$ the interval takes the form $\left(\underline{\theta}_{a}, \bar{\theta}_{a}\right)$. 
The next step is to look at the conditions preventing entry. Let $f\left(\mathbf{a}^{*}, i\right)$ be the number of distributions at which an entrant $i \notin P\left(\mathbf{a}^{*}\right)$ wins define $f^{*}=\max _{i \notin P\left(\mathbf{a}^{*}\right)} f\left(\mathbf{a}^{*}, i\right)$. If $f^{*}=0$ then entry is unprofitable. Thus, suppose $f^{*} \geq 1$. Then the no-entry condition must be satisfied both when the current distribution is such that the entrant would win and when it would not win. This condition is

$$
\max \left\{\theta+(1-\theta) \frac{f^{*}-1}{n-1},(1-\theta) \frac{f^{*}}{n-1}\right\} \leq c_{e}+c_{p} .
$$

The left hand side of (6) is minimized when $\theta=\frac{1}{n}$ for each $f^{*} \geq 1$, so a necessary condition for entry prevention is

$$
\frac{f^{*}}{n} \leq c_{e}+c_{p}
$$

Again, to have a sense of the values of $\theta$ that prevent entry it is useful to draw the left hand side of inequality (6) as a function of $\theta$.
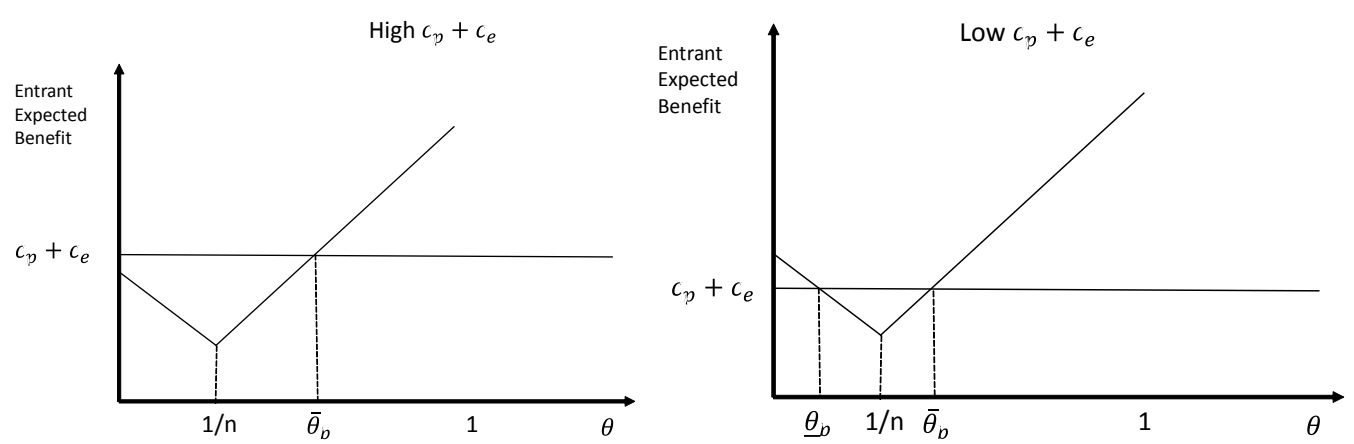

When $c_{p}+c_{e}$ is high $\left(c_{p}+c_{e}>\frac{f^{*}}{n-1}\right)$ then the interval of values of $\theta$ for which entry is not profitable is $\left[0, \bar{\theta}_{b}\right)$, while when the value of $c_{p}+c_{e}$ is lower (specifically, $\frac{f^{*}}{n} \leq c_{p}+c_{e}<\frac{f^{*}}{n-1}$ ) then the relevant interval takes the form $\left(\underline{\theta}_{b}, \bar{\theta}_{b}\right)$.

Notice that when $\theta=\frac{1}{n}$ then each distribution is equally likely and it does not matter which distribution occurred in the previous period. This is the best case scenario to maintain stability, because when $\theta \neq \frac{1}{n}$ some incumbents must have a lower expected utility than the one they get at $\theta=\frac{1}{n}$ under some current $\operatorname{mood} \mu_{k}$, while some potential entrants must have a higher expected utility. Since stability requires that the optimal action does not change for each realisation $\mu_{k}$, a uniform distribution over possible moods makes the conditions for stability easier to satisfy. 
Remark 1. Under reasonable assumptions on the evolution of $\theta$ as given by (1) - in particular that it does not depend on the current public opinion - it follows that each mood is expected to occur approximately a fraction $\frac{1}{n}$ of the periods in the 'future'. Thus, another interpretation of the conditions described in this section is that entry and exit should be best responses to the 'long-run distribution of moods'. We will return to this in greater detail in Section 4 .

\subsection{Stable Two Party Systems}

The Duverger's Law predicts that with a first-past-the-post electoral system, only two parties will carry a significant share of the votes. It is therefore interesting to investigate under what conditions, in our model, a stable twoparty system may emerge and what kind of positions can we expect the parties to occupy.

When two parties contest the election, the winner is always the party closer to the median of the realised distribution. Thus, if the two active parties are positioned at $x_{L}$ and $x_{R}$ with $x_{L}<x_{R}$, we can find a distribution $\mu_{i^{*}}$ with mean $m_{i^{*}}$ such that for each $i \leq i^{*}$ the left-wing party wins when the distribution is $\mu_{i}$ and vice-versa when $i>i^{*}$. The value $i^{*}$ is given by the highest $i$ such that $\frac{x_{L}+x_{R}}{2}>m_{i}$. Thus, for the two-party case, the necessary condition (5) becomes

$$
\min \left\{\frac{i^{*}}{n}, \frac{n-i^{*}}{n}\right\} \geq c_{p}
$$

The condition is more easily satisfied when both parties win approximately for the same number of distributions. In fact, the $i^{*}$ that maximizes the left hand side of (8) is $i^{*}=\frac{1}{n}\left\lfloor\frac{n}{2}\right\rfloor$. It is however important to note that the condition can be satisfied when $c_{p}$ is sufficiently low also for asymmetric party systems, i.e. systems in which one party tends to win more often than the other.

Consider next the possibility of entry. The main intuition can be obtained from the sequential entry model introduced by Palfrey [17]. In equilibrium the two parties cannot be positioned too close, since otherwise an entrant could enter on one side and win at least $50 \%$ of the times; only very high entry costs could prevent entry. If you call $q_{L}$ the highest number of cases in which an entrant to the left of $x_{L}$ wins, and similarly $q_{C}$ for someone entering in the interval $\left(x_{L}, x_{R}\right)$ and $q_{R}$ for someone entering to the right of $x_{R}$, then the obvious necessary condition is

$$
\max \left\{\frac{q_{L}}{n}, \frac{q_{C}}{n}, \frac{q_{R}}{n}\right\} \leq c_{e}+c_{p} .
$$


In section 5 we will discuss in greater depth the case of a two party system under a specific example.

One important observation that we can make now however is that outcomes in which the incumbent parties are at close positions require typically high entry cost. To see this, suppose that $n$ is even and the two incumbent parties are positioned at two consecutive positions in the interval $\left(m_{\frac{n}{2}}, m_{\frac{n}{2}+1}\right)$, i.e. they are positioned at the 'median of the medians' and each party wins for $\frac{n}{2}$ distributions. This implies $\min \left\{\frac{i^{*}}{n}, \frac{n-i^{*}}{n}\right\}=\frac{1}{2}$. Suppose $x_{L}$ is not the closest position to $m_{\frac{n}{2}}$ and $x_{R}$ is not the closest position to $m_{\frac{n}{2}+1}$. Then there are entrants on the right and on the left who can win for $\frac{n}{2}$ distributions, i.e. $\frac{q_{L}}{n}=\frac{q_{R}}{n}=\frac{1}{2}$, while $q_{C}=0$. Thus, condition (8) becomes $\frac{1}{2}>c_{p}$, while condition (9) becomes $\frac{1}{2}<c_{e}+c_{p}$. If $c_{e}=0$ then clearly the conditions cannot be simultaneously satisfied, so an equilibrium with ideologically close parties requires high cost of new political entry. As we have seen earlier, this is not true for two-party systems with ideologically distant parties.

\section{Long Run Dynamics}

Up to now we have discussed conditions for stability and characterised stable party systems. Specifically, we have shown that stability requires the perceived value of persistence $\theta_{e}$ of an ever changing public mood to remain in an interval around $\frac{1}{n}$, with the length of the interval determined by the participation $\operatorname{cost} c_{p}$ and the entry $\operatorname{cost} c_{e}$. As the perceived value of persistence becomes too high or (in some cases) too low, a stable party system falls apart and we start observing entry and exit activity.

Remark 2 (Continuity, equilibrium and stability). A particular configuration of active parties can continue to repeat itself (i.e., remain 'stable') in the short run even if $\theta_{e}$ goes out of the bounds so that it cannot be called a stable party system. This is because stability is a stronger requirement than Nash equilibrium: in a stable party system we must have the same Nash equilibrium for each realisation of $\mu$. A change in the estimated value of $\theta$ that makes the system unstable may still support the same system as a Nash equilibrium given the most recent realisation of public mood, though it ceases to be a Nash equilibrium for other moods.

It is now time to discuss the evolution of the persistence parameter $\theta$ over time and its implications for the dynamics of stable party systems. Thus the rest of the paper will address the second question we raised in Section 3: how is a party system sustained for a given period of time in the long run 
as a stable system when public mood is unpredictable and when does it get disturbed so that otherwise established democracies face the possibilities of transiting from one party system to another.

Fixed (known or unknown) persistence: The simplest and most obvious case is the one in which the parameter $\theta$ is fixed and known. When this is the case, the only link between periods is given by the fact that parties entering at the $t-1$ - th election do not have to pay the entry cost. Political configurations may change only when the political mood $\mu_{k}$ changes, but it is important to note that if a stable party configuration is achieved at any period, then there will be no further changes in the political system. On the other hand if $\theta$ is out of the interval for which a stable party system is possible, there always remains a strictly positive probability that next period mood swing is large enough to destroy the current Nash equilibrium, yielding a new set of active parties so that no stable party system can ever emerge.

The case in which $\theta$ is fixed but unknown is similar. Let $y_{t}$ be the random variable taking value 1 if the political mood remains the same at $t$ and zero otherwise. At the beginning of each period $T+1$ each party observes the realisation of $\frac{1}{T} \sum_{t=1}^{T} y_{t}$, a random variable that converges to $\theta$. Thus, in the long run, the parties learn $\theta$ and the party system converges to a stable configuration, if one exists for the true value of $\theta$ (if not, a stable party system cannot emerge).

Unknown and changing persistence: The most interesting and realistic case is the one in which $\theta$ is not observed and it is not fixed, but the sequence of political moods $\left\{\mu_{\tau}\right\}_{\tau=0}^{t}$ is observed by the parties before taking a decision at the beginning of period $t+1$. In such situations, the equilibrium political configurations may change even when the political mood does not change but expectations about the possibility of a change evolve. The rest of the paper will therefore pay special attention to an environment where public moods change through a Markov process with unknown and uncertain parameters.

\subsection{The Evolution of Persistence}

Suppose that the persistence parameter takes values from the set $\Theta=\left\{\theta_{1}, \ldots, \theta_{m}\right\}$ and the true current value is unknown to the current politicians. In order for them to decide whether to be active or not at time $t+1$, these politicians need to know the public mood $\mu^{t}$ prevailing at time $t$ and to form a probability distribution on $\Theta$, in particular an expected value $E\left[\theta^{t+1}\right]$. The question is what probability distribution to use to compute $E\left[\theta^{t+1}\right]$. 
We assume that $\theta^{t}$ changes over time following an irreducible Markov structure. The transition probability is given by the matrix

$$
\mathbf{P}=\left[\begin{array}{ccc}
p_{11} & \ldots & p_{1 m} \\
\ldots & \ddots & \ldots \\
p_{m 1} & \ldots & p_{m m}
\end{array}\right]
$$

where $p_{i j}=\operatorname{Pr}\left(\theta^{t+1}=\theta_{j} \mid \theta^{t}=\theta_{i}\right)$ and $\sum_{j=1}^{m} p_{i j}=1$ for each $i=1, \ldots, m$.

Politicians never observe the true value of $\theta$ but they do observe the value of a random variable $y_{t}$ that indicates whether at time $t$ the political mood changed or not. The distribution of $y_{t}$ conditional on $\theta^{t}=\theta_{i}$ is

$$
y_{t} \mid \theta_{i}=\left\{\begin{array}{cc}
1 & \text { with prob } \theta_{i} \\
0 & \text { with prob } \theta_{i}
\end{array}\right.
$$

The structure is therefore that of a hidden Markov chain. The evolution of beliefs on $\Theta$ can be represented as follows. Let

$$
\phi^{t}=\left[\begin{array}{c}
\phi_{1}^{t} \\
\vdots \\
\phi_{m}^{t}
\end{array}\right]
$$

be the vector of probabilities assigned at the end of time $t$ to different values of $\theta$, i.e. $\phi_{i}^{t}$ is the probability that the true value of the persistence parameter at time $t$ was $\theta^{t}=\theta_{i}$. This vector of probabilities can be computed from an initial distribution $\phi^{0}$ and a given history $h^{t}=\left(y_{1}, y_{2}, \ldots, y_{t}\right)$.

If $\phi^{t}$ is the probability distribution on $\theta^{t}$ conditional on history $h^{t}$, then the probability distribution on $\theta^{t+1}$ conditional on $h^{t}$ (that is, when no new observation of $y$ is added) is given by

$$
\widehat{\phi}^{t+1}=\mathbf{P}^{\prime} \phi^{t}
$$

or

$$
\widehat{\phi}_{i}^{t+1}=\sum_{j=1}^{m} p_{j i} \phi_{j}^{t} \quad i=1, \ldots, m .
$$

The distribution $\widehat{\phi}^{t+1}$ is the one used by the parties in evaluating whether or not to enter the electoral competition at the beginning of time $t+1$.

At the end of time $t+1$ the realisation of $y_{t+1}$ (i.e., whether or not the political mood at time $t+1$ is the same as at time $t$ ) is observed. We can 
therefore compute $\phi_{i}^{t+1}=\operatorname{Pr}\left(\theta^{t+1}=\theta_{i} \mid\left(h^{t}, y_{t+1}\right)\right)$. This is given by

$$
\begin{aligned}
\phi_{i}^{t+1} & =\operatorname{Pr}\left(\theta^{t+1}=\theta_{i} \mid y_{t+1}=y\right)=\frac{\operatorname{Pr}\left(y_{t+1}=y \mid \theta^{t+1}=\theta_{i}\right) \operatorname{Pr}\left(\theta^{t+1}=\theta_{i}\right)}{\sum_{j=1}^{m} \operatorname{Pr}\left(y_{t+1}=y \mid \theta^{t+1}=\theta_{j}\right) \operatorname{Pr}\left(\theta^{t+1}=\theta_{j}\right)} \\
& =\frac{\operatorname{Pr}\left(y_{t+1}=y \mid \theta^{t+1}=\theta_{i}\right) \widehat{\phi}_{i}^{t+1}}{\sum_{j=1}^{m} \operatorname{Pr}\left(y_{t+1}=y \mid \theta^{t+1}=\theta_{i}\right) \widehat{\phi}_{j}^{t+1}} .
\end{aligned}
$$

Now observe that

$$
\operatorname{Pr}\left(y_{t+1}=y \mid \theta^{t+1}=\theta_{i}\right)=\left\{\begin{array}{cl}
\theta_{i} & \text { if } y=1 \\
1-\theta_{i} & \text { if } y=0
\end{array}\right.
$$

so that, defining

$$
\widehat{E}\left[\theta^{t+1}\right]=E\left[\theta^{t+1} \mid h^{t}\right]=\sum_{j=1}^{m} \theta_{j} \widehat{\phi}_{j}^{t+1}
$$

we have

$$
\phi_{i}^{t+1}=\left\{\begin{array}{cl}
\frac{\theta_{i}}{\widehat{E}\left[\theta^{t+1}\right]} \widehat{\phi}_{i}^{t+1} & \text { if } y_{t+1}=1 \\
\frac{1-\theta_{i}}{1-\widehat{E}\left[\theta^{t+1}\right]} \widehat{\phi}_{i}^{t+1} & \text { if } y_{t+1}=0
\end{array}\right.
$$

At that point, the politicians will obtain a new probability distribution on $\theta^{t+2}$ as $\widehat{\phi}^{t+2}=\mathbf{P}^{\prime} \phi^{t+1}$, and they will use $\widehat{\phi}^{t+2}$ for the entry or exit decision at the beginning of time $t+2$.

We will be particularly interested in the values taken by the sequence $\left\{\widehat{\phi}^{t}, \widehat{\phi}^{t+1}, \ldots, \widehat{\phi}^{t+T}\right\}$ when the political mood does not change, i.e. when $y_{t+1}=y_{t+1}=\ldots=y_{t+T}=1$. Define $\boldsymbol{\theta}^{\prime}=\left[\theta_{1}, \ldots, \theta_{m}\right]$ and let

$$
\mathbf{D}=\left[\begin{array}{ccc}
\theta_{1} & \ldots & 0 \\
0 & \ddots & 0 \\
0 & \ldots & \theta_{m}
\end{array}\right]
$$

be the associated diagonal matrix which has $\theta_{i}$ in position $(i, i)$ and zero elsewhere. The updating procedure in the case $y_{t+1}=1$ can be compactly summarised as follows:

- Start with $\widehat{\phi}^{t}$, the probability distribution on $\theta^{t}$ given history $h_{t-1}$; 
- Compute $\phi^{t}$, the probability distribution on $\theta^{t}$ given history $h_{t}$, assuming that $y_{t}=1$. This is given by

$$
\phi^{t}=\frac{1}{\boldsymbol{\theta}^{\prime} \widehat{\phi}^{t}} \mathbf{D} \widehat{\phi}^{t}
$$

- Compute $\widehat{\phi}^{t+1}$, the probability distribution on $\theta^{t+1}$ given history $h_{t}$. This is given by

$$
\widehat{\phi}^{t+1}=\mathbf{P}^{\prime} \phi^{t}
$$

The transition from $\widehat{\phi}^{t}$ to $\widehat{\phi}^{t+1}$ when the political mood does not change at time $t$ can therefore be written compactly as

$$
\widehat{\phi}^{t+1}=\frac{1}{\boldsymbol{\theta}^{\prime} \widehat{\phi}^{t}} \mathbf{P}^{\prime} \mathbf{D} \widehat{\phi}^{t}
$$

or equivalently as

$$
\widehat{\phi}_{i}^{t+1}=\frac{\sum_{j=1}^{m} p_{j i} \theta_{j} \widehat{\phi}_{j}^{t}}{\sum_{j=1}^{m} \theta_{j} \widehat{\phi}_{j}^{t}} \quad i=1, \ldots, m
$$

Depending on the assumptions made on the transition matrix $\mathbf{P}$ we will observe different dynamics of beliefs. We will introduce assumptions ensuring some monotonicity properties in the dynamics that we next study.

\subsection{Two possible degrees of persistence}

Before we move to the general case it is useful to consider the case $m=2$, i.e. $\Theta=\left\{\theta_{1}, \theta_{2}\right\}$. In this case all we have to do is to track the evolution of a single number, for example $\widehat{\phi}_{1}^{t}$. From (12) we have:

$$
\widehat{\phi}_{1}^{t+1}=\frac{p_{11} \theta_{1} \widehat{\phi}_{1}^{t}+p_{21} \theta_{2}\left(1-\widehat{\phi}_{1}^{t}\right)}{\theta_{1} \widehat{\phi}_{1}^{t}+\theta_{2}\left(1-\widehat{\phi}_{1}^{t}\right)}
$$

Define the function

$$
\beta(\phi)=\frac{p_{11} \theta_{1} \phi+p_{21} \theta_{2}(1-\phi)}{\theta_{1} \phi+\theta_{2}(1-\phi)}
$$

and observe that $\beta$ is continuous, differentiable, strictly positive at $\phi=0$ and strictly lower than 1 at $\phi=1$. The derivatives are:

$$
\beta^{\prime}(\phi)=\frac{\left(p_{11}-p_{21}\right) \theta_{1} \theta_{2}}{\left(\phi \theta_{1}+(1-\phi) \theta_{2}\right)^{2}} \quad \beta^{\prime \prime}(\phi)=\frac{2 \theta_{1} \theta_{2}\left(\theta_{2}-\theta_{1}\right)\left(p_{11}-p_{21}\right)}{\left(\phi \theta_{1}+(1-\phi) \theta_{2}\right)^{3}}
$$


so their signs do not depend on $\phi$ on the interval $[0,1]$ and it is equal for the first and second derivative. Given that $p_{i i}+p_{i j}=1$, there can be only two possible cases: $^{6}$

1. Weak inertia $\left(p_{11}<p_{21}\right)$ : This is the case of over-volatile persistence of public moods where there is a unique solution to the equation $\phi_{1}^{*}=$ $\beta\left(\phi_{1}^{*}\right)$. The movement of the belief when the political mood does not change can be represented through the following diagram.

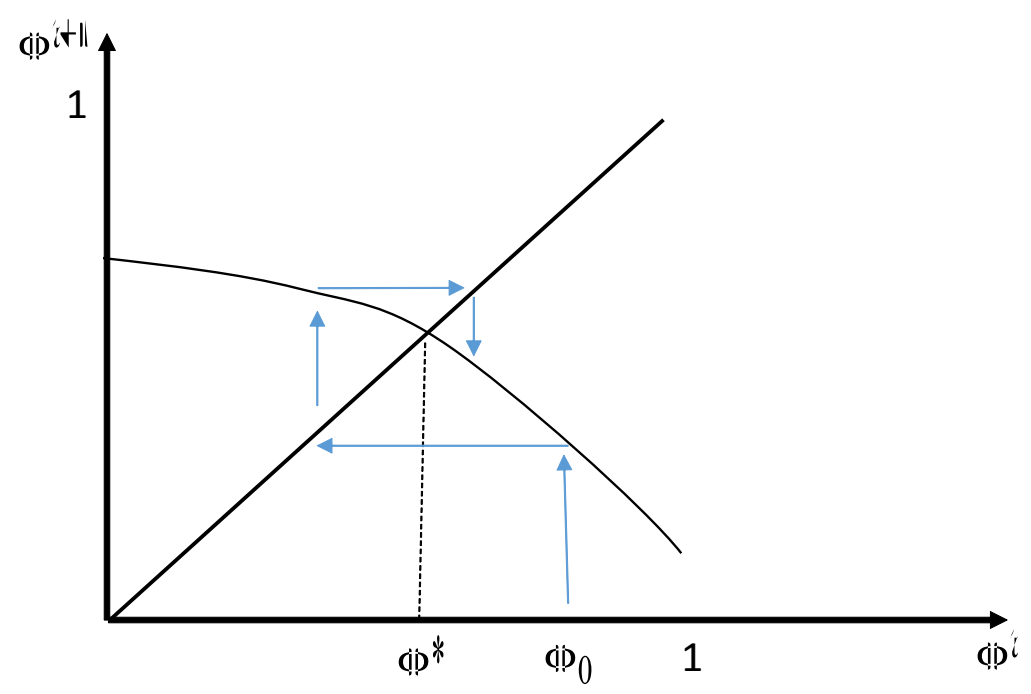

If there is convergence, it will not be monotone. Rather, the belief will jump above $\phi_{1}^{*}$ whenever it is below and vice-versa. Thus when persistence of public mood is more likely to change than be sustained, the politicians' beliefs may converge but through a period of strong fluctuation around the point of convergence.

2. Strong inertia $\left(p_{11}>p_{21}\right)$ : In this case current persistence in public moods is less likely to change and in principle, the equation $\phi_{1}^{*}=\beta\left(\phi_{1}^{*}\right)$ might have multiple solutions (and we shall generalise this in Section 4.3

\footnotetext{
${ }^{6}$ Excluding the trivial case $p_{21}=p_{11}$, which implies a constant belief.
} 
through Assumption 2).

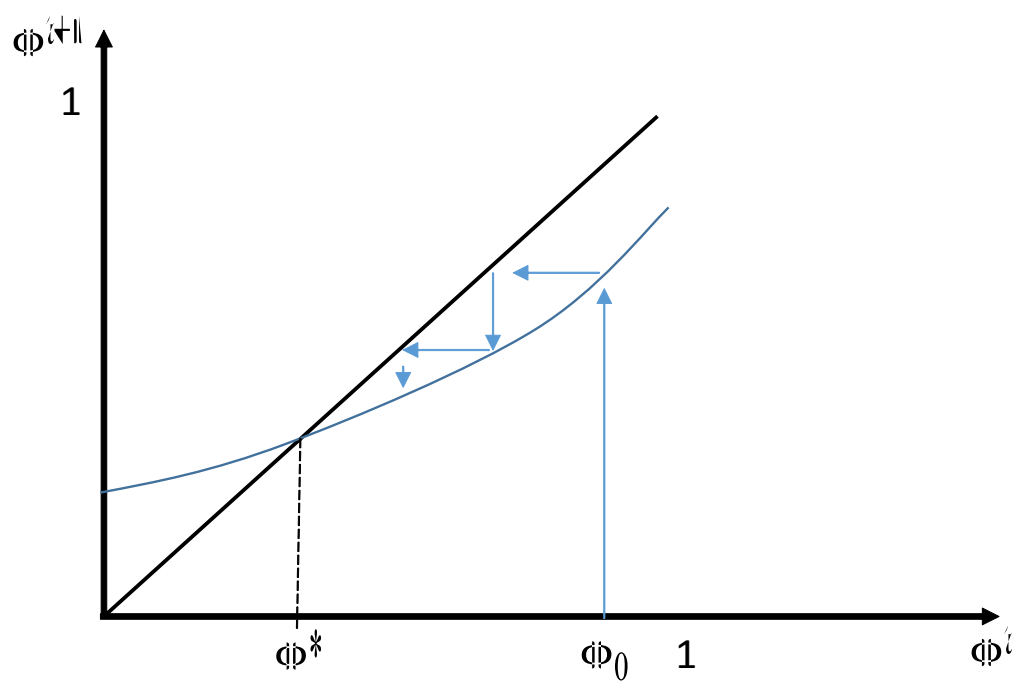

However, since the function $\beta$ is strictly convex and $\beta(1)<1$, the solution is indeed unique. Moreover, the convergence is in fact monotone: if $\phi_{1}$ starts above $\phi_{1}^{*}$ then it decreases in every period and it reaches asymptotically $\phi_{1}^{*}$.

While the central theme of the paper is to study the impact of volatility in public moods on electoral outcomes, we take the stand that this volatility is of a first order nature and not of higher order where the very persistence parameter can itself become stochastically too volatile. Hence we will be particularly interested in the case of strong inertia. Our stand is also motivated by the fact that with strong inertia, beliefs move in the intuitive direction, meaning that the 'perceived' probability of a realisation of the higher value of the persistence parameter actually increases when more persistence in the public opinion is observed.

So given strong inertia in the persistence parameter, consider a stable party system requiring the perceived persistence to fall within an interval $(\underline{\theta}, \bar{\theta})$. Define $\theta_{e}^{*}=\theta_{1} \phi_{1}^{*}+\theta_{2}\left(1-\phi_{1}^{*}\right)$, where $\phi_{1}^{*}$ is the solution to $\phi_{1}=\beta\left(\phi_{1}\right)$ and suppose $\theta_{e}^{*}>\bar{\theta}$. Then, with strong inertia, a sufficiently long sequence of periods with the same political mood will increase the estimate $\theta_{e}$ up to a point at which $\theta_{e}>\bar{\theta}$, thus destabilising the party system. This has an important implication in our understanding of eventual instability of a party system that experiences a long period of repetition. It shows that when the politicians do not know the true parameter that determines the probability of a change in public opinions but are aware of strong inertia in this probability, then an initially stable party system must eventually break down if 
the society does not undergo a change in public mood during this initial phase and the highest possible value of the persistence parameter falls outside the interval of the perceived value required for stability to be sustained. It therefore highlights an interesting and somewhat counter-intuitive point: a stable history of public opinions may not be conducive to the stability of the party system; rather, a stable party system is less likely to change if the public opinion would have some amount of realised volatility.

\subsection{The general case}

We now study the general case with $m \geq 2$ possible values of the persistence parameter $\theta$. Our analysis will focus on the case in which beliefs exhibit a monotone dynamics, i.e. whenever the distribution does not change the beliefs put more weight on higher values of $\theta$. For a given matrix $\mathbf{P}$ and for $r=1, \ldots, m$, define

$$
F_{j}(r)=\sum_{i=1}^{r} p_{j i},
$$

the cumulative distribution of the probability on $\Theta$ conditional on the current state being $\theta_{j}$. Following the analysis for the $m=2$ case, define the mapping $\boldsymbol{\beta}: \Delta \Theta \rightarrow \Delta \Theta$ as

$$
\boldsymbol{\beta}(\phi)=\frac{1}{\boldsymbol{\theta}^{\prime} \boldsymbol{\phi}} \mathbf{P}^{\prime} \mathbf{D} \phi
$$

The set $\Delta \Theta$ can be endowed with the partial order $\succsim$ given by first order stochastic dominance. We are interested in conditions on the mapping $\boldsymbol{\beta}$ that make it increasing, i.e. whenever $\phi^{\prime} \succsim \phi$, then $\boldsymbol{\beta}\left(\phi^{\prime}\right) \succsim \boldsymbol{\beta}(\phi)$. We now introduce an assumption which turns out to be necessary and sufficient to ensure that $\boldsymbol{\beta}$ is increasing.

Assumption 2. For each $k$ and $q$ with $k>q$ the inequality

$$
\left(\theta_{k}-\theta_{q}\right) F_{j}(r) \geq \theta_{k} F_{k}(r)-\theta_{q} F_{q}(r)
$$

holds for each $r \in\{1, \ldots m\}$ and for each $j \in\{1, \ldots m\}$.

A few observations are in order. Inequality (14) is always satisfied when $r=m$, since in that case $F_{i}(m)=1$ for each $i$. For the case $m=2$, the assumption reduces to $p_{11} \geq p_{21}$, which is the condition that we found previously (this can be easily checked because the only relevant cases involve $k=2$ and $q=r=1$ ). Finally, Assumption 2 implies that the distributions $F_{i}(\cdot)$ must be first-order stochastically increasing in $i$. To see this, notice that, by taking $j=q$, inequality (14) becomes

$$
F_{q}(r) \geq F_{k}(r), \quad r \in\{1, \ldots m\} .
$$


Thus, Assumption 2 is stronger than the requirement that the conditional distributions are increasing in the state. This actually implies that we do not have to check (14) for each $j$. Instead, since $F_{j}(r)$ is decreasing in $j$, it sufficient that

$$
\left(\theta_{k}-\theta_{q}\right) F_{m}(r) \geq \theta_{k} F_{k}(r)-\theta_{q} F_{q}(r)
$$

for each $r$. We can now state a preliminary result.

Proposition 4. The mapping $\boldsymbol{\beta}(\boldsymbol{\phi})$ defined by (13) is increasing if and only if Assumption (2) holds.

The mapping $\boldsymbol{\beta}: \Delta \Phi \rightarrow \Delta \Phi$ is continuous and defined on a compact space, so a fixed point exists. However, just knowing that a fixed point exists does not provide much information on the dynamics of beliefs. As shown for the case $m=2$ and $p_{11}<p_{21}$, beliefs may move non-monotonically and convergence may not occur. On the other hand, if $\boldsymbol{\beta}$ is increasing as well, then we have additional results that allow us to draw conclusions on the dynamics of beliefs and therefore on the dynamics of party systems. This observation leads us to the main result of this section.

Proposition 5. If Assumption (2) holds the set of fixed points of $\boldsymbol{\beta}$ is nonempty and it has a smallest and largest element. Let $\phi_{l}$ be the smallest fixed point of $\boldsymbol{\beta}$ and let $\theta_{e}^{l}$ be the expected value of $\theta$ under $\phi_{l}$. If a stable party system $\left(\mathbf{a}^{*}, \Theta^{*}\right)$ is such that $\sup \Theta^{*}<\theta_{e}^{l}$ then a sufficiently long sequence of unchanged distributions will destabilise the system.

Proposition 5 generalises the intuition obtained from looking at the $m=2$ case. If the mapping $\boldsymbol{\beta}$ is increasing the expected value of persistence increases when more persistence is observed, at least if the initial estimated persistence is not too high. A stable party system will typically be destabilised when the political mood does not change and the same party keeps winning elections. At some point some of the losers will exit or some entry will occur. The fact that a long-enough run of unchanged moods that keeps increasing the perceived value of the persistence parameter can make active losers exit is easy to see as eventually the prospects of victory for these losing parties will dwindle and fail to sustain the costs of running the party. But why can such a run of stable public moods lead to an eventuality where new parties enter? To see this note that the active configuration of parties can be such that the party that has been winning all along the recent history of stable public opinion would lose if the sustained public mood was more favourable for a party that is currently sitting out of the race. When there is repeated realisation of the current mood, the perceived persistence can move up significantly, thereby providing room for this currently inactive party to 
enter and cover the costs of doing so. Thus a long run of stability of public mood can destroy stability of the party system and bring in a new party.

\section{An Example of Stable Party Systems with Gaussian Distributions}

The analysis in Section 4.1 has characterised conditions under which one would expect stable party systems to be sustained or to be destabilised. While the dynamics are straightforward and less interesting with fixed or known persistence, in a world where the politicians do not observe a stochastically evolving persistence, long run stability of stable party systems is more likely when the public mood undergoes periodic changes as otherwise stability can be broken. Keeping these results on the dynamics of stable party systems in the background, we now work out an example to see how stable party systems can be computed.

The example we set involves a policy space given by the real line $(-\infty,+\infty)$ and five possible moods $\mu_{i}, i=1, \ldots, 5$. Each mood has a normal distribution with standard deviation $\sigma=1$ and mean $m_{i}$. We choose these distributions such that

$$
m_{1}=0.2, \quad m_{2}=0.4, \quad m_{3}=0.6, \quad m_{4}=0.8 \quad \text { and } \quad m_{5}=1 .
$$

We do not describe fully the feasible policy set and hence the exact identities or the number of parties. It is convenient to think that any point in the real line can be a party, with the obvious approximations for finite sets when we look at the equilibria discussed below.

With 5 possible distributions, stable party systems will have at most 5 parties. The number and ideological identities of the active parties will of course depend on the parameters. In what follows we fully characterise the set of parameters for which a one-party stable system exists. We then look at some examples of 2-party stable systems. It should at that point be clear how to build 3, 4 and 5-party stable systems.

\subsection{Stable One-Party Systems}

Since we have 5 moods, Proposition 3 implies that the necessary and sufficient condition for the existence of a stable one-party system is $c_{e}+c_{p}>\frac{2}{5}$. In fact, it is clear that no matter where a single party is located, there is one entrant who can win in at least two cases, so whenever $c_{e}+c_{p}<\frac{2}{5}$ no stable one-party system is possible. When $c_{e}+c_{p}>\frac{2}{5}$ then stable one-party systems can be characterised as follows: 
- Moderate cost of entry $\left(\frac{2}{5}<c_{e}+c_{p}<\frac{3}{5}\right)$ : In this case the equilibrium is unique. The single party occupies the position closest to $m_{3}=0.6$ that the feasible policy set admits, a 'median of median voters' result. Entry to the left or to the right can yield victory at most in 2 of the possible moods, so it is not profitable. To find the range of values of $\theta$ for which this configuration can be sustained, observe that an entrant located sufficiently close to the incumbent will win with probability $\frac{1}{2}(1-\theta)$ if not located on the thinner side of the current distribution given the current winner's position, and with probability $\theta+(1-\theta) \frac{1}{2}$ otherwise. The two inequalities $\frac{1}{2}(1-\theta) \leq c_{e}+c_{p}$ and $\theta+\frac{1}{4}(1-\theta) \leq c_{e}+c_{p}$ yield $\Theta^{*}=\left[\max \left\{0,1-2\left(c_{e}+c_{p}\right)\right\}, \frac{4}{3}\left(c_{e}+c_{p}\right)-\frac{1}{3}\right]$. For example, if $c_{e}+c_{p}=\frac{1}{2}$ the range is $\left[0, \frac{1}{3}\right]$.

- High cost of entry $\left(\frac{3}{5}<c_{e}+c_{p}<\frac{4}{5}\right)$ : In this case entry is prevented as long as the potential entrant is able to win in at most 3 of the possible moods. Thus the incumbent can be located anywhere between the closest point to $m_{2}=0.4$ and the closest point to $m_{4}=0.8$ that the feasible policy set admits. If the incumbent is located at the closest point to $m_{3}$ then the analysis of the previous case applies. If the incumbent is located at the closest point to $m_{2}$ or $m_{4}$ then the two relevant inequalities for entry prevention are $\frac{3}{5}(1-\theta) \leq c_{e}+c_{p}$ and $\theta+\frac{1}{2}(1-\theta) \leq c_{e}+c_{p}$ that together yield $\Theta^{*}=\left[0,2\left(c_{e}+c_{p}\right)-1\right]$.

- Very high cost of entry $\left(\frac{4}{5}<c_{e}+c_{p}<1\right)$ : In this case entry is prevented as long as the entrant is able to win in at most in 4 cases. Thus the incumbent can be located anywhere between the closest point to $m_{1}=0.2$ and the closest point to $m_{5}=1$. When the incumbent is located in the interval between $m_{2}$ and $m_{4}$ then the analysis of the two previous points applies. If the incumbent is located at the closest point to $m_{1}$ or $m_{5}$ then the two relevant inequalities for entry prevention are $\frac{4}{5}(1-\theta) \leq c_{e}+c_{p}$ and $\theta+\frac{3}{4}(1-\theta) \leq c_{e}+c_{p}$ yielding $\Theta^{*}=$ $\left[0,4\left(c_{e}+c_{p}\right)-3\right]$.

Summary: As expected, the length of $\Theta^{*}$ increases with $c_{e}+c_{p}$. Moreover if $c_{e}$ and $c_{p}$ are such that a stable one-party system exists, then higher entry costs allow for a more extremist party.

\subsection{Stable Two-Party Systems}

For two-party systems we have to check that the incumbents are willing to stay, as well as that no entrant can get in. With an odd number of moods, 
in a two-party system with contesting profile of platforms $\left\{x_{L}, x_{R}\right\}$ there will be a party winning for a fewer number of distributions - the 'less dominant party'. If this party does not exit, then the more dominant one will not as well. Let $k$ be the number of moods in which the less-dominant party wins. Then $k \in\{1,2\}$. The condition for continued participation is as follows. When the party does not win under the current distribution, the probability of victory in the next election is $\frac{k}{4}(1-\theta)$. Otherwise, the probability of victory is $\theta+\frac{k-1}{4}(1-\theta)$. Thus, the condition is

$$
\min \left\{\frac{k}{4}(1-\theta), \theta+\frac{k-1}{4}(1-\theta)\right\} \geq c_{p} .
$$

If $c_{p} \geq \frac{k}{5}$ then the condition cannot be satisfied. When $\frac{k-1}{4} \leq c_{p} \leq \frac{k}{5}$ then the condition is satisfied for $\theta \in\left(\frac{4}{5-k} c_{p}-\frac{k-1}{5-k}, 1-\frac{4}{k} c_{p}\right)$, and when $c_{p} \leq \frac{k-1}{4}$ the condition is satisfied for $\theta \in\left[0, \frac{4}{5-k} c_{p}-\frac{k-1}{5-k}\right)$.

Consider now the conditions implied by entry prevention. Suppose that the highest number of distributions at which an entrant can win is $f$. The expected payoff from entry is therefore $\frac{f}{4}(1-\theta)$ if the current distribution is not one under which the entrant would win and it is $\theta+\frac{f-1}{4}(1-\theta)$ if the entrant would win under the current distribution. Thus the condition to prevent entry is

$$
\max \left\{\frac{f}{4}(1-\theta), \theta+\frac{f-1}{4}(1-\theta)\right\} \leq c_{e}+c_{p} .
$$

The condition cannot be satisfied if $c_{e}+c_{p}<\frac{f}{5}$, i.e. whenever $c_{e}+c_{p}<\frac{f}{5}$ any configuration $\left\{x_{L}, x_{R}\right\}$ which allows an entrant to win under $f$ distributions is not a stable system. If $\frac{f}{5} \leq c_{e}+c_{p} \leq \frac{f}{4}$ then the condition is satisfied for $\theta \in\left(1-\frac{4}{f}\left(c_{e}+c_{p}\right), \frac{4}{5-f}\left(c_{e}+c_{p}\right)-\frac{f-1}{5-f}\right)$. Finally, if $c_{e}+c_{p} \geq \frac{f}{4}$ the condition is satisfied for $\theta \in\left[0, \frac{4}{5-k}\left(c_{e}+c_{p}\right)-\frac{k-1}{5-k}\right)$.

We can now use the results above to establish whether any given pair $\left\{x_{L}, x_{R}\right\}$ is a stable party system. If we define $x^{*}=\frac{x_{L}+x_{R}}{2}$ then the positions of the two parties can be written as $x_{L}=x^{*}-\varepsilon$ and $x_{R}=x^{*}+\varepsilon$ for some $\varepsilon>0$. The question then becomes whether a pair $\left(x^{*}, \varepsilon\right)$ can be a stable party system. Notice that $x^{*}$ determines which one is the 'dominant' party (the party winning most of the times) while $\varepsilon$ is a measure of the ideological distance between the two parties.

Suppose for example that $x^{*}=0.3$. In this case the party located at $x_{L}$ wins only under distribution $\mu_{1}$, so that $k=1$. Thus, the first condition for this to be a stable party system is $c_{p}<\frac{1}{5}$. The values of $\varepsilon$ which are allowed are determined by the no-entry condition. Entry may occur to the 
left of $x^{*}-\varepsilon$, in the interval $\left(x^{*}-\varepsilon, x^{*}+\varepsilon\right)$ or to the right of $x^{*}+\varepsilon$ and for each distribution $\mu_{i}$ we can compute the distribution of votes for the entrant and the incumbent parties. Let $v_{E}^{i}$ be the share of the vote of the entrant under distribution $\mu_{i}$ and define $v_{L}^{i}, v_{R}^{i}$ to be the vote shares of the incumbent parties. Let $\Phi$ be the cumulative distribution of a standard normal. When entry occurs on the left we have

$$
v_{E}^{i}=\Phi\left(\frac{x^{*}-\varepsilon+x_{E}}{2}-m_{i}\right), v_{L}^{i}=1-v_{R}^{i}-v_{E}^{i} \text { and } v_{R}^{i}=1-\Phi\left(x^{*}-m_{i}\right),
$$

and similar formulas can be used for entry at the center and at the right. We compute possible equilibria for $c_{p}+c_{e} \leq \frac{1}{5}$ and $\frac{1}{5} \leq c_{p}+c_{e} \leq \frac{2}{5}$; the other cases are similar.

- Low cost of entry $\left(c_{e}+c_{p} \leq \frac{1}{5}\right)$ : In this case stability requires that no entrant is able to win under any distribution. Consider the following ranges of $\varepsilon$ :

a) $\varepsilon \leq 0.2$. In this case an entrant at $x^{*}-\varepsilon$ can win if the distribution is $\mu_{1}$. Thus there is no stable system.

b) $0.2 \leq \varepsilon \leq 1.0081$. In this case an entrant at $x^{*}+\varepsilon$ can win if the distribution is $\mu_{5}$. Thus there is no stable system.

c) $1.0081 \leq \varepsilon$. In this case an entrant located at 0.4 wins when the distribution is $\mu_{1}$. Thus there is no stable system.

We conclude that there is no value of $\varepsilon$ such that a stable party system with $x^{*}=0.3$ exists.

- Moderate cost of entry $\left(\frac{1}{5} \leq c_{p}+c_{e} \leq \frac{2}{5}\right)$ : In this case stability requires that no entrant is able to win under any distribution. Consider the following ranges of $\varepsilon$ :

a) $\varepsilon \leq 0.897$. In this case an entrant at $x^{*}+\varepsilon$ can win if the distributions are $\mu_{4}$ and $\mu_{5}$. Thus there is no stable system.

b) $0.897 \leq \varepsilon \leq 0.933$. In this case no entrant can win for two distributions. Thus, for this range of values, there is a stable system.

c) $0.933 \leq \varepsilon$. In this case there is an entry at the centre ensuring victory for at least two distributions. For example, if $\varepsilon=1$ then entry at 0.3 ensures victory under $\mu_{1}$ and $\mu_{2}$. Thus there is no stable system. 
We conclude that for each $\varepsilon \in(0.897,0.933)$ there is a stable two-party system with $x^{*}=0.3$.

Summary: In general, if the parties are ideologically too close ( $\varepsilon$ is low) then entry either on the left or the right is profitable, while if they are too far away ( $\varepsilon$ is large) then entry at the centre becomes profitable. So the cost of entry $c_{p}+c_{e}$ has to be large enough to make sure that for some intermediate value of $\varepsilon$ neither entry on the wing nor entry at the centre are profitable. When costs are very low (the case $c_{e}+c_{p} \leq \frac{1}{5}$ ) this is impossible. When costs start to grow (the case $\frac{1}{5} \leq c_{e}+c_{p} \leq \frac{2}{5}$ ) then intermediate values of $\varepsilon$ such that entry is never profitable appear. As the value of $c_{e}+c_{p}$ increases, the set of values of $\varepsilon$ generating stable two-party systems expands.

\section{Extensions and Conclusion}

In order to keep the model tractable and the results sharp, we have made some strong assumptions. It is worth discussing, albeit briefly, what happens when the assumptions are relaxed.

We have assumed that politicians care only about the next election. What if they have a longer horizon? If the assumption is that the party leader remains in charge for a fixed number $n$ of periods then the analysis is similar. Younger leaders will be tempted to enter more, since entering today lowers more the total cost of running when the tenure is longer. The main complication however is that at any given date, leaders at different points in their careers will have different incentives to enter, so the equilibrium will depend on the age distribution of available leaders. A variant of the model may contemplate the possibility that a leader remains at the helm of the party until it is defeated in an election. In that case a party leader will typically be more reluctant to enter, since entering today implies not only paying a cost but also removal from leadership in case of loss. Thus, by entering a leader forfeits the option value of waiting for the next elections, when conditions may be more favorable.

Another simplifying assumption we made is that the party identity is fixed, as in the standard citizen candidate framework. This can be relaxed, assuming that at each time $t$ the current initial platform is $x_{i t}$ and the platform can be moved in an interval $\left(x_{i t}-\delta, x_{i t}+\delta\right)$ around $x_{i t}$, maybe at a cost that depends on the distance from the initial platform. In this case platforms will be changed to follow the popular mood and we can expect less entry to occur in the long-run with respect to the model with fixed identity.

Finally, we have assumed that the probability of change in mood does not depend on the policy implemented. It is sometimes claimed (e.g. Schlesinger 
[21]) that changes in the public mood are more likely when the same policy $x$ is implemented for a long period of time, since the problems of the policy become more apparent. This would be more complicated to deal with, as it would require the evolution of $\theta$ to depend on the equilibrium history. 


\section{Appendix}

Proof of Proposition 1. Let $\left(\mathbf{a}^{*}, \Theta^{*}\right)$ be a stable party system. Since $\mathbf{a}^{*}$ is a strict Nash equilibrium, the set $\Theta^{*}$ cannot be singleton. This follows from the fact that if $\mathbf{a}^{*}$ is a strict Nash equilibrium for each $\mu \in \mathcal{M}$ at some $\widehat{\theta}_{e}$ then it must remain a strict Nash equilibrium for an appropriate interval around $\widehat{\theta}_{e}$. Now consider two elements $\theta^{(1)}$ and $\theta^{(2)}$ in $\Theta^{*}$. Since the utility functions are linear in $\theta_{e}$ and actions are strictly preferred, the vector $\mathbf{a}^{*}$ remains a strict Nash equilibrium for any linear combination of $\theta^{(1)}$ and $\theta^{(2)}$.

Proof of Proposition 2. Suppose $\theta_{e}>1-c_{p}$ and let $\mu^{t-1}=\mu_{k}$. Let $\mathbf{a}^{*}$ be a strict Nash equilibrium and let $P\left(\mathbf{a}^{*}\right)$ be the set of entering parties. The cardinality of $P\left(\mathbf{a}^{*}\right)$ must be one. This is because only one party can win when the distribution is $\mu_{k}$ and the utility of a party that does not win when the political mood is $\mu_{k}$ is bounded above by

$$
\mathbf{V}_{j}\left(\phi,\left(a_{j}, \mathbf{a}_{-j}\right), a_{j}^{t-1}, \mu_{k}\right) \leq\left(1-\theta_{e}\right)-c_{p} .
$$

The right hand side is strictly negative when $\theta_{e}>1-c_{p}$, thus contradicting the fact that entry is optimal.

Suppose now that $\theta_{e}>\max \left\{1-c_{p}, c_{p}+c_{e}\right\}$ If the only entrant $i^{*}$ is such that $\left|m_{k}-x_{i^{*}}\right|$ is not minimized over $X$, then there is a party $j$ such that $\left|m_{k}-x_{j}\right|<$ $\left|m_{k}-x_{i^{*}}\right|$. If party $j$ enters then it wins the election when the mood is $\mu_{k}$. Thus, a lower bound on its expected utility is $\theta_{e}-\left(c_{e}+c_{p}\right)$, which is assumed to be strictly positive. Finally, it is immediate to see that when the only entrant minimizes the distance from $m_{k}$ then the expected utility of any other entrant would be negative.

Proof of Proposition 3. We first prove necessity. Consider a single party $i^{*}$ located at $x^{*} \in\left[m_{q}, m_{q+1}\right]$ and suppose that $x^{*}$ is the closest position to $m_{q}$ (the cases in which $x^{*}$ is not the closest position to $m_{q}$ is similar). For this to be a stable party system it has to be true that no entry is possible just to the right and just to the left of $x^{*}$ for every distribution. The expected value of entry for the party that just precedes $x^{*}$ when the current state is $\mu_{k}$ with $k \leq q-1$ is

$$
l_{i}^{\theta}=\theta+(1-\theta) \frac{q-2}{n-1} .
$$

This is because the party wins when the mood remains the same (probability $\theta$ ) and when the mood changes (probability $1-\theta$ ) and one of the remaining $q-2$ distribution with a median lower than $m_{q}$ is drawn. When $\mu_{k}$ is such that $k \geq q$ then the expected value of entry is

$$
l_{i}^{1-\theta}=(1-\theta) \frac{q-1}{n-1} .
$$

Thus, a necessary condition to prevent entry from the left is

$$
c_{e}+c_{p}>\max \left\{l_{i}^{\theta}, l_{i}^{1-\theta}\right\} .
$$

Since $\max \left\{l_{i}^{\theta}, l_{i}^{1-\theta}\right\}$ is minimized at $\theta=\frac{1}{n}$, this implies that a necessary condition is

$$
c_{e}+c_{p}>\frac{q-1}{n} \text {. }
$$


A similar reasoning regarding entry on the right gives

$$
c_{e}+c_{p}>\frac{n-q}{n} .
$$

Thus, the necessary condition for the existence of a one-party stable system is

$$
c_{e}+c_{p}>\max \left\{\frac{n-q}{n}, \frac{q-1}{n}\right\} .
$$

for some $q$. The RHS of (15) is minimized at $q=\frac{n+1}{2}$ if $n$ is odd and at $q \in\left\{\frac{n}{2}, \frac{n}{2}+1\right\}$ if $n$ is even. It thus follows that a necessary condition is

$$
c_{e}+c_{p}>\frac{1}{n}\left\lfloor\frac{n-1}{2}\right\rfloor .
$$

To prove sufficiency, consider a situation in which $n$ is odd and a party system in which only the party located at the value $x^{*}$ that minimizes the distance from $m_{\frac{n+1}{2}}$. If $\theta=\frac{1}{n}$ then entry is not profitable for each $\mu_{k}$. Since all the relevant inequalities are strict, there is an interval around $\theta=\frac{1}{2}$ for which this is a stable party system. The upper bound of the interval must be strictly greater than $\frac{1}{n}$, while the lower bound must be strictly less.

Proof of Proposition 4. We first prove necessity. Let

$$
\boldsymbol{\beta}(\phi)=\frac{1}{\boldsymbol{\theta}^{\prime} \phi} \mathbf{P}^{\prime} \mathbf{D} \phi
$$

If $\boldsymbol{\beta}$ is increasing, than whenever $\mathbf{f} \succsim \mathbf{g}$ we have $\boldsymbol{\beta}(\mathbf{f}) \succsim \boldsymbol{\beta}(\mathbf{g})$, i.e.

$$
\sum_{i=1}^{r} \boldsymbol{\beta}_{i}(\mathbf{f}) \leq \sum_{i=1}^{r} \boldsymbol{\beta}_{i}(\mathbf{g})
$$

for each $r \in\{1, \ldots, m\}$, where

$$
\boldsymbol{\beta}_{i}(\mathbf{f})=\frac{\sum_{j=1}^{m} p_{j i} \theta_{j} f_{j}}{\sum_{j=1}^{m} \theta_{j} f_{j}} .
$$

Consider a pair $(\mathbf{f}, \mathbf{g})$ such that

$$
f_{i}=\left\{\begin{array}{ccc}
g_{i} & \text { if } & i \notin\{q, k\} \\
g_{i}-\varepsilon & \text { if } & i=q \\
g_{i}+\varepsilon & \text { if } & i=k
\end{array}\right.
$$

for two indices $k>q$ and $\varepsilon \in\left(0, \min \left\{g_{q}, 1-g_{k}\right\}\right)$. Since $\mathbf{f} \succsim \mathbf{g}$ (it is obtained by transferring probability $\varepsilon$ from state $\theta_{q}$ to the larger state $\theta_{k}$ ), inequality (16) requires

$$
\frac{\sum_{j=1}^{m}\left(\sum_{i=1}^{r} p_{j i}\right) \theta_{j} g_{j}+\varepsilon\left(\left(\sum_{i=1}^{r} p_{k i}\right) \theta_{k}-\left(\sum_{i=1}^{r} p_{q i}\right) \theta_{q}\right)}{\sum_{j=1}^{m} \theta_{j} g_{j}+\varepsilon\left(\theta_{k}-\theta_{q}\right)} \leq \frac{\sum_{j=1}^{m}\left(\sum_{i=1}^{r} p_{j i}\right) \theta_{j} g_{j}}{\sum_{j=1}^{m} \theta_{j} g_{j}}
$$

for each $r \in\{1, \ldots, m\}$. Using $F_{j}(r)=\sum_{i=1}^{r} p_{j i}$ and rearranging terms, we can rewrite (17) as

$$
\sum_{j=1}^{m}\left(\theta_{k}\left(F_{j}(r)-F_{k}(r)\right)-\theta_{q}\left(F_{j}(r)-F_{q}(r)\right)\right) \theta_{j} g_{j} \geq 0
$$


The only way in which this can be true for each distribution $g$ is that, for each pair $k$ and $q$ with $k>q$ the inequality

$$
\left(\theta_{k}\left(F_{j}(r)-F_{k}(r)\right)-\theta_{q}\left(F_{j}(r)-F_{q}(r)\right)\right) \geq 0
$$

holds for each $j \in\{1, \ldots, m\}$ and $r \in\{1, \ldots, m\}$. Rearranging terms we can write inequality (18) as

$$
\left(\theta_{k}-\theta_{q}\right) F_{j}(r) \geq \theta_{k} F_{k}(r)-\theta_{q} F_{q}(r) .
$$

To prove sufficiency, observe that if $\mathbf{f} \succsim \mathbf{g}$ then $\mathbf{f}$ can be obtained from $\mathbf{g}$ in a finite number of steps by subtracting probability from a lower state and adding it to a higher state.

Proof of Proposition 5. Consider the set $\Delta \Theta$ endowed with first-order stochastic dominance order $\succsim$. Then $(\Delta \Theta, \succsim)$ is a complete lattice. Since $\beta: \Delta \Theta \rightarrow \Delta \Theta$ is increasing, we can apply Tarski's fixed point theorem for increasing functions (Tarski [22]; see Vives [23] for an exposition) and conclude that the set of fixed points of $\boldsymbol{\beta}$ is not empty and it is in fact a complete lattice. Thus, is has a largest and a smallest element. Call $\phi_{l}$ the lowest fixed point of $\boldsymbol{\beta}$ and let $\theta_{e}^{l}$ be the expectation of $\theta$ taken under $\phi_{l}$.

Define now recursively the function $\boldsymbol{\beta}^{\mathbf{n}}(\boldsymbol{\phi})$ as

$$
\begin{aligned}
& \boldsymbol{\beta}^{\mathbf{0}}(\phi)=\phi \\
& \boldsymbol{\beta}^{\mathbf{n}}(\phi)=\boldsymbol{\beta}\left(\boldsymbol{\beta}^{\mathbf{n}-\mathbf{1}}(\phi)\right) \text { for } n \geq 1 .
\end{aligned}
$$

Thus, $\boldsymbol{\beta}^{\mathbf{n}}(\phi)$ is the belief held on $\Theta$ when the initial belief is $\phi$ and a sequence of $n$ elections without changing mood is observed.

If $\left(\mathbf{a}^{*}, \Theta^{*}\right)$ is a stable party system then $\Theta^{*}$ is an interval; let $\bar{\theta}=\sup \Theta^{*}$ and assume $\bar{\theta}<\theta_{e}^{l}$.

Consider a distribution $\phi$ for which the expected value of $\theta$ under $\phi$ is in the interval $\Theta^{*}$. Let $\phi$ be the lowest element of $\Delta \Theta$, i.e. the distribution putting probability 1 on $\theta_{1}$, the lowest element of $\Theta$. Then $\phi \succsim \underline{\phi}$ and since $\boldsymbol{\beta}$ is increasing we have $\boldsymbol{\beta}^{\mathbf{n}}(\phi) \succsim \boldsymbol{\beta}^{\mathbf{n}}(\underline{\phi})$ for each $n$. Furthermore, since $\boldsymbol{\beta}(\underline{\phi}) \succsim \underline{\phi}$ we have $\boldsymbol{\beta}^{\mathbf{n}}(\underline{\phi}) \succsim \boldsymbol{\beta}^{\mathbf{n}-1}(\underline{\phi})$ for each $n$, so the sequence $\left\{\boldsymbol{\beta}^{\mathbf{n}}(\phi)\right\}_{n=1}^{\infty}$ is increasing. It therefore converges to a fixed point of $\boldsymbol{\beta}$. Thus, there is $n$ sufficiently large such that the expectation of $\theta$ taken under $\boldsymbol{\beta}^{\mathbf{n}}(\phi)$ is strictly greater than $\bar{\theta}$. Since $\boldsymbol{\beta}^{\mathbf{n}}(\phi) \succsim \boldsymbol{\beta}^{\mathbf{n}}(\underline{\phi})$ for each $n$, the expectation of $\theta$ taken under $\boldsymbol{\beta}^{\mathbf{n}}(\phi)$ is also strictly greater than $\bar{\theta}$. 


\section{References}

[1] Paul R. Abramson, John H. Aldrich, Phil Paolino and David W. Rohde (1995) 'Third-Party and Independent Candidates in American Politics: Wallace, Anderson, and Perot', Political Science Quarterly, 110 (3): 349 - 367.

[2] James Adam, M. Clark, L. Ezrow and G. Glasgow (2004) 'Understanding change and stability in party ideologies: do parties respond to public opinion or to past election results?' British Journal of Political Science, $34,589-610$.

[3] Stefano Bartolini and Peter Mair (1990) 'Identity, Competition and Electoral Availability: The Stabilisation of European Electorates, 1885 1985', Cambridge: Cambridge University Press.

[4] Timothy Besley and Stephen Coate (1997) 'An Economic Model of Representative Democracy', Quarterly Journal of Economics, 112: 85-114.

[5] Sandro Brusco and Jaideep Roy (2011) 'Aggregate Uncertainty in the Citizen Candidate Model Yields Extremist Parties', Social Choice and Welfare, 36 (1): 83-104.

[6] Ian Budge (1994) 'A New Theory of Party Competition: Uncertainty, Ideology and Policy Equilibria Viewed Comparatively and Temporally', British Journal of Political Science, 24, 443-67.

[7] Davidson D. Byers and David A. Peel (1997) 'Modelling Political Popularity: an analysis of long range dependence in opinion poll series', Journal of Royal Statistical Society, Series A, 160: 471 - 490.

[8] Suzanna DeBoof (2000) 'Persistence and Aggregation of survey data over time: from microfoundations to macropersistence', Electoral Studies, 19: $9-29$.

[9] John Duggan and Jean Guillaume Forand (2013) 'Markovian Elections', working paper.

[10] John Duggan and César Martinelli (2014) 'The Political Economy of Dynamic Elections: A Survey and Some New Results', working paper.

[11] Timothy Feddersen, Itai Sened and Stephen Wright (1990) 'Rational Voting and Candidate Entry under Plurality Rule', American Journal of Political Science, 34: 1005-16. 
[12] Joseph Greenberg and Kenneth Shepsle (1987) 'The Effect of Electoral Rewards in Multiparty Competition with Entry', American Political Science Review, 81: 525 - 538.

[13] Simon Hug (2001) 'Altering Party Systems: Strategic Behavior and the Emergence of New Political Parties in Western Europe', Ann Arbor: University of Michigan Press.

[14] M. Lebo, R.W. Walker and H. D. Clarke (2000) 'You must remember this: dealing with long memory in political analysis', Electoral Studies, 19: $31-48$.

[15] Samuel Merrill III, Bernard Gorfman and Thomas L. Brunell (2008) 'Cycles in American National Electoral Politics, 1854-2006: Statistical Evidence and an Explanatory Model', American Political Science Review, 102 (1): 1-17.

[16] Martin Osborne and Al Slivinski (1996) 'A Model of Political Competition with Citizen Candidates', Quarterly Journal of Economics, 111: 65-96.

[17] Thomas Palfrey (1984) 'Spatial Equilibrium with Entry', Review of Economic Studies, LI: 139-156.

[18] James Stimson (1999) Public Opinion in America: Moods, Cycles and Swings, $2^{\text {nd }}$ edition. Boulder: Westview Press.

[19] James Stimson (2004) Tides of Consent: How Public Opinion Shapes American Politics, Cambridge: Cambridge University Press.

[20] James Stimson (2012) Website: http://www.unc.edu/ cogginse/Policy_Mood.html

[21] Arthur M. Schlesinger Jr. (1986) 'The Cycles of American History', Boston: Houghton Mifflin Company.

[22] Alfred Tarski (1955) 'A Lattice-Theoretical Fixed Point Theorem and its Applications', Pacific Journal of Mathematics, 5: 285-308.

[23] Xavier Vives (1999) Oligopoly Pricing. Old Ideas and New Tools, MIT Press.

[24] Christopher Wlezien (2000) 'An essay on 'combined' time series processes', Electoral Studies, 19: 77 - 93. 